\title{
Sawtooth-like x-ray emission observed in EBIT
}

\author{
R. Radtke ${ }^{\mathrm{a} *}$, C. Biedermann ${ }^{\mathrm{a}}$, and P. Bachmann ${ }^{\mathrm{a}}$ \\ ${ }^{a}$ Max-Planck-Institut für Plasmaphysik, Bereich Plasmadiagnostik, EURATOM Association, D-10117 \\ Berlin, Germany
}

The evolution of a mixture of highly charged Ar and Ba ions was measured in an electron beam ion trap (EBIT) by recording the characteristic x-ray emission from trapped ions. A special feature in the spectra are sawtooth-like intensity variations caused by a periodic collapse of the ion inventory in the trap. The effect requires favorable conditions to become present and is very sensitive to the trapping conditions. Analysis of the measurements is based on a time-dependent calculation of the trapping process. Simulations show that sawtooth activity results from the feedback between the low- $Z$ Ar and high- $Z$ Ba ions (Hopf bifurcation). Sawtooth spectra open up a spectroscopic method to test theoretical EBIT models and probe the dynamics in ion traps and sources.

\section{Introduction}

An important area in electron beam ion traps and sources is the evolution of ions and much effort has been directed to generate data on this process [1-8]. It is fundamental to the understanding of trapping conditions in EBIT itself and for designing experiments, extending from x-ray spectroscopy of highly charged ions to using the trap inventory as a source for external experiments. Theoretical calculations using computer models provide the majority of data from which the evolution of charge balance and ion temperature are derived. However, the predictive quality of the results is limited because of uncertainties in the atomic physics data. In addition, often approximations are needed in the modelling physics to make the calculations tractable. Measurements of the evolution of ions were carried out at early as well as late confinement times. The emphasis in the experiments was on determining the ions' development toward equilibrium $[2,5-7]$ and on providing trapping lifetimes for confined ions $[1,8]$.

In what follows we present measurements and calculations of the evolution of an ensemble of highly charged $\operatorname{Ar}(Z=18)$ and $\mathrm{Ba}(Z=56)$

\footnotetext{
*accepted for publication in Nuclear Instruments \& Method in Physical Research: Section B, conference proceedings to $11^{\text {th }}$ International Conference on the Physics of Highly Charged Ions (HCI-2002) Sep. 1-6, 2002, Caen, France
}

ions in an EBIT. Working with light ions along with heavier ones resembles what is termed evaporative cooling in electron beam ion traps and sources [9]. This technique permits EBIT to produce and store a much higher number of highly charged ions than would be possible without cooling. The present study relies on an unusual variation of evaporation cooling; the rate at which the light component is injected into the trap was much larger than the influx of the heavy component. Operating EBIT in this way, we could observe sawtooth-like $\mathrm{x}$ emission caused by a periodic collapse of the ion inventory in the trap. To our knowledge, sawtooth activity in an EBIT has not been measured previously nor has it been predicted by theoretical calculations. The effect requires favorable conditions to become present, and the waveform of the sawtooth spectrum is very sensitive to the trapping conditions. These unique properties suggest the possibility of using sawtooth spectra as benchmark data to test theoretical EBIT models.

\section{Experiment}

In our experiment, neutral argon gas was continuously fed into the trap from an injector while barium is an EBIT-intrinsic contaminant evaporated from the heated cathode of the electron gun. EBIT was operated in a static mode where all levels of the applied voltages and the beam current 
were held constant as a function of time for a single measurement. The beam energy was fixed throughout the measurements at $E_{b}=5.0 \mathrm{keV}$ limiting the maximum charge of Ar to 18+ and of $\mathrm{Ba}$ to $46+$. Characteristic x-ray spectra from the confined ions were measured for $25 s$ after closing the trap at $t=0$. We have monitored the radiation in the region above $\sim 500 \mathrm{eV}$ with a solidstate Ge detector. Data from the detector were stored in an analog-to-digital convertor (ADC) and fed into a multichannel scaler (MCS). The latter recorded the event time for the observed $\mathrm{x}$ rays with a resolution of $50 \mathrm{~ms}$. Time profiles of x-ray emission were constructed by combining the pulse-height spectrum from the ADC with the MCS data, creating a scatter plot of x-ray events versus time, applying cuts along separate $\mathrm{x}$-ray bands and projecting the x-ray events found in these cuts onto the time axis. In the present study, analysis of the $\mathrm{x}$ emission was done for the collisionally excited 3.1-keV-energy $\operatorname{Ar} n=2-1$ and 4.8-keV-energy Ba $n=3-2$ x rays.

We have measured time profiles as a function of electron-beam current and axial trap depth $V_{\text {trap }}$. The results of the $V_{\text {trap }}$ scan are presented in Fig. 1. $V_{\text {trap }}=V_{\text {bias }}+V_{i p}$ is the sum of the upper drift tube's bias $(-25 \mathrm{~V}<$ $V_{\text {bias }}<300 \mathrm{~V}$ ) and the image potential formed by the electron beam and the geometry of the trap $V_{i p} \simeq 0.14 \times\left(I_{b} \sqrt{m_{e}} / \epsilon_{0} \sqrt{E_{b}}\right)$. Most prominent in Fig. 1 is the change in the functional form of the profiles when $V_{\text {trap }}$ is varied. For a shallow trap (2 $V$ axial potential) the Ar ions are lost essentially immediately after being created while the intensity of the Ba ions increases and levels to a plateau. As the trap is made deeper a significantly greater amount of Ar ions is confined which interact with an increasing number of $\mathrm{Ba}$ ions. In the $4-V$ plot, a situation is shown where a threshold is reached for the feedback between the two components, evidenced by small oscillations in the $\mathrm{Ar}$ and $\mathrm{Ba}$ intensity at the early confinement times. The growth of the feedback as $V_{t r a p}$ is further raised is seen in the $9-V$ and $15-V$ plots. In the $15-V$ plot sawtooth-like signatures manifest in the spectrum with the signal for $\mathrm{Ar}(\mathrm{Ba})$ decreasing (increasing) over successive time periods. Towards the end of each period, a sudden

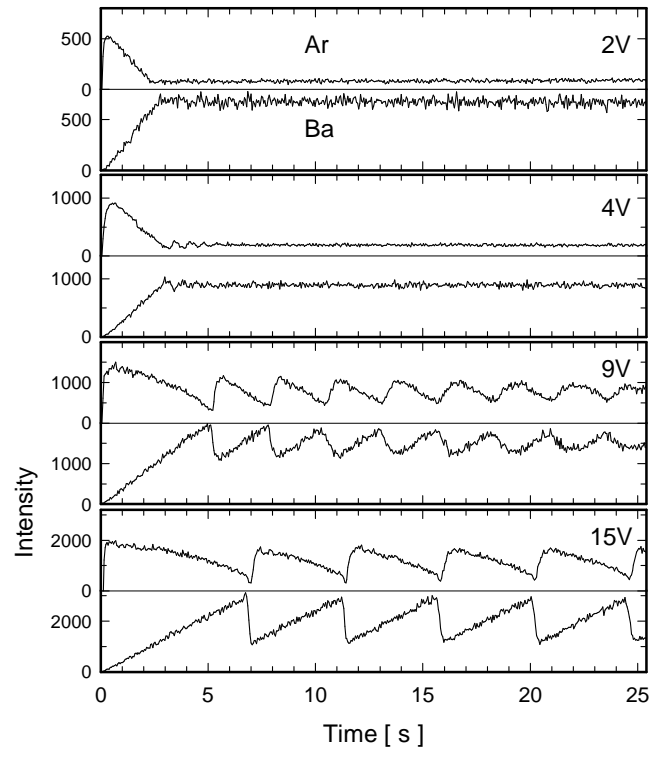

Figure 1. Time profile of $n=2-1$ and $n=3-2$ emission spectra of $\mathrm{Ar}$ and $\mathrm{Ba}$ for different trap depths $V_{\text {trap }}$. The value of $V_{\text {trap }}$ is plotted in each panel showing the Ar trace. The beam current and energy are fixed parameters $\left(I_{b}=50 \mathrm{~mA}\right.$, $\left.E_{b}=5.0 \mathrm{keV}\right)$.

decline in the intensity is detected indicating that the ion inventory has collapsed in the trap. The collapse occurs on a time scale of $\sim 100 \mathrm{~ms}$ and restores the conditions for a new start-up of the process. It should be noted that the effect even persisted for ten minutes, the longest exposure used in our investigation.

\section{Calculation}

In order to provide an explanation for the sawtooth mechanism, simulations of the evolution of $\mathrm{Ar}$ and $\mathrm{Ba}$ ions have been carried out for conditions similar to those used in the experiment. The modelling procedure included: (a) electronimpact ionization and (b) electron-beam heating of the ions, (c) ion-ion energy exchange, (d) axial ion escape from the trap, (e) radial overlap factors (electron-ion and ion-ion), and (f) trap neutral- 
ization by ions. The evolution of the ion density is described by

$$
\frac{d n_{q}}{d t}=\frac{n_{q-1}}{\tau_{q-1}^{i o n}}-\frac{n_{q}}{\tau_{q}^{i o n}}-\frac{n_{q}}{\tau_{q}^{c o n}},
$$

where $n_{q}$ is the density of trapped ions (Ar or Ba) in charge state $q, \tau_{q-1}^{i o n}$ the characteristic time to create the charge state $q$ and $\tau_{q}^{c o n}$ the ion confinement time. Charge creation is calculated using the ionization cross sections devised by Lotz [10]. For $\tau_{q}^{c o n}$ we used the definition from Ref. [11]

$\tau_{q}^{c o n}=\frac{\sqrt{2}}{3} \frac{w_{q} \exp \left(w_{q}\right)}{\sum_{p} \nu_{q p}}$,

with $w=\frac{q e V_{a x}}{k T_{q}}$ and where $\nu_{q p}$ is the collision rate between the $q$ th and $p$ th ionic species

$\nu_{q p}=\frac{4}{3} \sqrt{2 \pi} n_{p}\left(\frac{q p e^{2}}{M_{q}}\right)^{2}\left(\frac{M_{q}}{k T_{q}}\right)^{3 / 2} \ln \Lambda_{q p}$.

$T_{q}$ and $M_{q}$ are the temperature and mass of the $q$ th ionic species and $\Lambda_{q p}$ is the ion-ion Coulomb logarithm. The ion temperature is obtained from the balance between heating and cooling taking the processes (b), (c) and (d) into account:

$$
\frac{d}{d t}\left(\frac{3}{2} k T_{q} n_{q}\right)=\frac{d W_{q}^{b}}{d t}-\frac{d W_{q}^{d}}{d t}+\sum_{p}\left(\frac{d W_{q}^{c}}{d t}\right)_{p} .
$$

For the energies $W_{q}^{b, c, d}$ we have employed the expressions [11]:

$\frac{d W_{q}^{b}}{d t}=\frac{4 \pi}{3} n_{q} \frac{j_{e}}{e} \frac{q^{2} e^{4}}{E_{b}} \frac{m}{M_{q}} \ln \Lambda_{q e}$,

$\frac{d W_{q}^{d}}{d t}=\frac{n_{q}}{\tau_{q}^{c o n}}\left(q e V_{a x}+k T_{q}\right)$,

$\left(\frac{d W_{q}^{c}}{d t}\right)_{p}=2 \nu_{q p} n_{q} \frac{M_{q}}{M_{p}} \frac{k\left(T_{p}-T_{q}\right)}{\left(1+M_{q} T_{p} / M_{p} T_{q}\right)^{3 / 2}}$.

Here $j_{e}$ is the electron-beam current density, $m$ the electron mass and $\ln \Lambda_{q e}$ the electron-ion Coulomb logarithm. The sum in Eqs. (2) and (4) is over all $\mathrm{Ar}$ and $\mathrm{Ba}$ charge states in question. The effect of trap neutralization was incorporated by adding the ion potential $V_{i o n}$ to the trap depth: $V_{a x}=V_{\text {trap }}+V_{\text {ion }}$. Values of $V_{\text {ion }}$ were calculated
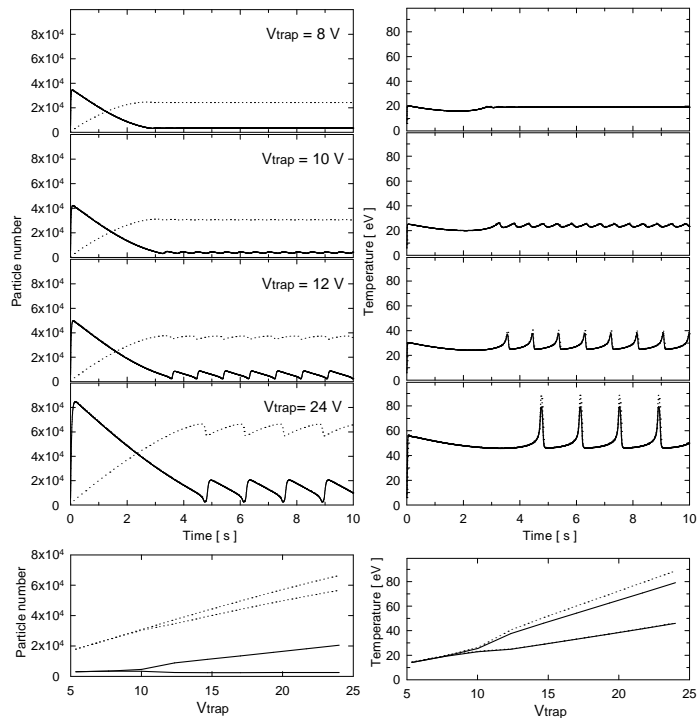

Figure 2. Calculated evolution of Ar (solid line) and $\mathrm{Ba}$ (dashed line) ions. The graph shows the total number of trapped ions $N_{A r}$ and $N_{B a}$ (left) and the ion temperature of $A r^{18+}$ and $\mathrm{Ba}^{46+}$ (right). The value of $V_{\text {trap }}$ is plotted in the panel showing the particle number. Beam current and energy are as for Fig. 1. The calculations are based on Eqs. (1)-(8) and assume $n_{A r, 0}=$ $2.2 \times 10^{5} \mathrm{~cm}^{-3}$ and $n_{B a, 0}=2.6 \times 10^{3} \mathrm{~cm}^{-3}$. The bottom plots show the attractors of ion number and temperature in dependence of $V_{\text {trap }}$; above $\approx 8 \mathrm{~V}$ the two-component ion plasma begins to bifurcate (Hopf bifurcation).

from the predicted density and characteristic radius $r_{q}$ of the confined ions assuming constant ion-density distributions for $r<r_{q}\left(r_{q}\right.$ was evaluated from the ion-ion overlap factors)

$V_{i o n}=-\sum_{q} \frac{e n_{q} q r_{q}^{2}}{4 \epsilon_{0}}\left(1+2 \ln \left(\frac{r_{\text {trap }}}{r_{q}}\right)\right)$.

$r_{\text {trap }}=0.5 \mathrm{~cm}$ is the radius of the center drift tube. The coupled non-linear rate equations were solved by a FORTRAN code using stiff stable NAG routines[12]. To speed up the calculation and identify certain trends in the results, the 
problem of modelling $18+46=64$ different ions was reduced to only 7 representatives of each component: $q=1,2,5,10,16,17,18$ for $\mathrm{Ar}$ and $q=1,3,6,10,23,38,46$ for Ba. These states were selected to cover the time scale for low as well as highly charged ion production, extending from $10^{-6} s$ to $1 s$. We stress that covering the complete time scale is essential to warrant the dynamics of ion creation; picking only the highest charge states of $\mathrm{Ar}$ and $\mathrm{Ba}$ did not enable us to simulate any oscillations. We calculated time profiles $n_{q}(t)$ for $\mathrm{Ar}$ and $\mathrm{Ba}$ ions in dependence of $V_{t r a p}$ and $n_{A r, 0}$ and $n_{B a, 0}$, the densities of neutrals in the beam. $n_{A r, 0}$ and $n_{B a, 0}$ can only roughly be estimated in the experiment. With regard to the condition $n_{B a, 0} \ll n_{A r, 0}$ we have adjusted $n_{A r, 0}$ and $n_{B a, 0}$ in the calculation until qualitatively similar behavior to the experimental profiles was observed for a range of $V_{\text {trap }}$ values. The results are shown in Fig. 2.

\section{Discussion}

The calculations accurately predict the time scale of the collapsing ion inventory and indicate when the system bifurcates. As expected, the effect becomes present at increased density when the $\mathrm{Ar}$ and $\mathrm{Ba}$ ions are well mixed by collisions. The sawteeth appear according to the following scheme: Ba ions accumulate in the trap at slightly increasing temperature until the benefit of cooling is reduced due to the reduction of $N_{A r}$. This accelerates the temperature increase and particle loss from the trap, such that $N_{B a}$ becomes a decreasing function of time. The temperature increases further as $N_{B a}$ (and $N_{A r}$ ) falls, and thus the ion-ion collision rate $\left(\nu_{i} \propto n_{i} / T_{i}^{3 / 2}\right)$ is strongly reduced. This decouples the twocomponent system (note the different temperatures for $\mathrm{Ar}$ and $\mathrm{Ba}$ during the collapse) allowing the Ar ions to invade the trap and stay in the beam longer. They are heated less by the electron beam and cool the $\mathrm{Ba}$ ions at declining temperature until $N_{B a}$ becomes sufficiently high that the competition between Ar and Ba starts again.

The calculations did not accurately reproduce the tooth lengths measured or the bifurcation of the particle number as a function of $V_{\text {trap }}$. This underlines the complexity of the ion dynamics in EBIT, which is difficult to predict with simple assumptions. It is likely that the origin of this discrepancy is related to the problem of finding the potential $V_{a x}$ deformed by the trapped ions and that an improvement in $V_{a x}$ determination could resolve the difference. Variations of axial trapping potential result in Hopf bifurcation with fairly simple dynamics; more complicated dynamics appear when varying other parameters (e.g. densities) making even chaotic phenomena possible.

A detailed discussion of the sawtooth spectra is the subject of a forthcoming paper. We would like to thank G. Fussmann for many stimulating discussions during the course of this work.

\section{REFERENCES}

1. M.B. Schneider, M.A. Levine, C.L. Bennett, J.R. Henderson, D.A. Knapp, and R.E. Marrs, in International Symposium on Electron Beam Ion Sources and Their Application, edited by A. Hershcovitch (Conf. Proc. No. 188, AIP, New York, 1989), p. 158.

2. B.M. Penetrante, J.N. Bardsley, D. DeWitt, M. Clark, and D. Schneider, Phys. Rev. A 43(1991) 4861.

3. B.M. Penetrante, J.N. Bardsley, M.A. Levine, D.A. Knapp, R.E. Marrs, Phys. Rev. A 43 (1991) 4873.

4. I.V. Kalagin, D. Küchler, V.P. Ovsyannikov, and G. Zschornack, Plasma Sources Sci. Technol. 7 (1998) 441.

5. C. Biedermann, T. Fuchs, P. Liebisch, R. Radtke, E. Behar, and R. Doron, Physica Scripta T80 (1999) 303.

6. J.V. Porto, I. Kink, and J.D. Gillaspy, Rev. Sci. Instrum. 71 (2000) 3050.

7. F.J. Currell, H. Kuramoto, S. Ohtani, C. Scullion, E.J. Sokell, and H. Watanabe, Physica Scripta T92 (2001) 147.

8. C. Biedermann,T. Fuchs, G. Fussmann, and R. Radtke, in Trapping Highly Charged Ions: Fundamentals and Applications, edited by J. Gillaspy (Nova Science Publishers, Inc., New York, 2001), p. 80.

9. M.A. Levine, R.E. Marrs, J.R. Henderson, 
D.A. Knapp, and M.B. Schneider, Physica Scripta T22 (1998) 157.

10. W. Lotz, Z. Phys. A 216 (1998) 241.

11. G. Fussmann, C. Biedermann, and R. Radtke, in Advanced Technologies Based on Wave and Beam Generated Plasmas, edited by $\mathrm{H}$. Schlüter and A. Shivarova (Kluwer Academic Publishers, 1999), p.429.

12. Numerical Algorithm Group, <http://www.nag.co.uk/numeric/Fortran_Libraries.asp>. 